\section{UNA EXPERIENCIA DE INNOVACIÓN DOCENTE EN EL ÁMBITO UNIVERSITARIO. USO DE LAS NUEVAS TECNOLOGÍAS}

\author{
M. ${ }^{a}$ Dolores Lerís López \\ y M. ${ }^{a}$ Luisa Sein-Echaluce Lacleta \\ Departamento de Matemática Aplicada. \\ Edificio Torres Quevedo del Campus Río Ebro, 50018-Zaragoza \\ dleris@unizar.es \\ mlsein@unizar.es
}

\section{AN EDUCATIONAL INNOVATION EXPERIENCE IN THE UNIVERSITY FIELD. THE USE OF NEW TECHNOLOGIES}

\begin{abstract}
Several teaching initiatives and experiments, carried out by the authors, are presented in this paper. By means of Moodle (an e-learning platform) as a support to on-site teaching, they were applied to some Mathematics subjects of Engineering degrees at the University of Zaragoza. The learning projects, started up over the last few years, have been influenced by the context in which they have been applied. Therefore, we will indicate the strengths and weaknesses which have been detected as influential factors in the design of the courses. Furthermore, our experiments are presented with an explanation of the design of the courses, the methodology and the activities used in the learning units of which they consist as well as of the way to carry out the proactive and reactive tutorial tasks in a blended-learning environment.
\end{abstract}

KEY WORDS: Blended learning; cooperative learning: mathematics.

\section{INTRODUCCIÓN}

Las nuevas tecnologías forman parte en mayor o menor medida de nuestras vidas y naturalmente han entrado de lleno en la actividad docente. La mayoría de los profesores universitarios, quizás todos, hemos ido utilizando unas $u$ otras herramientas tecnológicas en los proyectos docentes de nuestras asignaturas. Todos tenemos experiencia y nosotras queremos ofrecer la nuestra a través de este artículo presentando una aplicación de las nuevas tecnologías en un entorno universitario estándar, las lecciones que hemos aprendido y también los éxitos que hemos alcanzado. El camino recorrido no ha sido recto, directo, ha habido desánimo por los fallos o por las imprevisiones y ha habido mucha satisfacción por los logros alcanzados; hasta aquí hemos llegado y hoy pensamos, hacemos
RESUMEN: En este artículo exponemos algunas de las iniciativas docentes y experiencias que hemos llevado a cabo las autoras en algunas asignaturas de Matemáticas de titulaciones de Ingenieria de la Universidad de Zaragoza y dentro del ámbito de la enseñanza presencial con ayuda de la plataforma electrónica de aprendizaje Moodle. Los proyectos de aprendizaje que hemos ido poniendo en marcha durante estos últimos años han estado condicionados por el contexto en el que se están aplicando, por ello indicamos aquí las fortalezas y debilidades que hemos detectado como factores influyentes en el diseño de los cursos. Asimismo, presentamos nuestras experiencias explicando el diseño de los cursos, la metodología y actividades que usamos en las unidades de aprendizaje que los conforman y la forma en que hemos puesto en marcha tutorias proactivas y reactivas en el entorno de aprendizaje semipresencial.

PALABRAS CLAVE: Aprendizaje semipresencial; aprendizaje cooperativo; matemáticas.

y decimos lo que aquí estamos escribiendo. Seguimos dispuestas a avanzar.

Vamos a escribir sobre novedades docentes; debemos matizar: sobre novedades en el sentido de que no son prácticas habituales en las universidades españolas. Dos fuerzas están impulsado nuestro movimiento durante esta última década: por un lado, el extraordinario desarrollo de las herramientas tecnológicas dedicadas al aprendizaje y, por otro, la convergencia hacia un Espacio Europeo de Educación Superior (en adelante, EEES).

Desarrollamos nuestra labor docente en la Universidad de Zaragoza (en adelante, UZ). Esta Universidad española, como muchas otras, apostó por crear un entorno de docencia virtual que se viene denominando Anillo Digital 
Docente (en adelante, ADD). El servicio de docencia virtual que ofrece la UZ promueve su uso como apoyo a la docencia de las asignaturas regladas de los planes de estudio vigentes y sustenta la docencia a distancia de cursos que se comparten con otras universidades españolas constituyendo el denominado G9.

Aunque inicialmente, desde 2002-2003, la única plataforma de e-learning que sustentaba el ADD era WebCT, ya a partir de 2007-08 se instaló también la plataforma Moodle; así pues, hoy día el ADD de la UZ tiene dos esqueletos tecnológicos: WebCT y Moodle. En nuestro caso, hemos optado por utilizar Moodle desde el momento en que fue puesta en servicio en la UZ, poniendo así nuestra experiencia previa en el uso de esa plataforma al servicio de la práctica docente reglada.

La convergencia hacia el EEES supone una revolución en las metodologías docentes tradicionalmente utilizadas en la Universidad española. Tenemos que dejar de pensar y hablar de enseñanza para llegar a pensar y hablar de aprendizaje. No es una tarea fácil, en absoluto, pues hemos de reconocer que la mayoría de los profesores que vamos a poner en marcha los nuevos planes de estudio hemos sido educados en el modelo de aprendizaje pasivo y ése es el que nos resulta más fácil de reproducir. En nuestro caso, hemos iniciado el proceso de adaptación al EEES de modo que, gracias a algunos excelentes cursos de formación (Bara y Valero, 2007, y Valero, 2009) y a las mejoras de medios materiales (más ordenadores y aulas para los usuarios, mejor tecnología, etc.), hemos incorporado distintas metodologías de aprendizaje. El proceso de convergencia hacia el EEES nos ha obligado a poner en nuestro punto de mira dos asuntos: por un lado, delimitar los objetivos de aprendizaje en términos de competencias genéricas y específicas $y$, por otro, reflexionar y estudiar cómo aprenden nuestros estudiantes y cuáles son las metodologías adecuadas para los nuevos objetivos; la consecuencia ha sido la puesta en marcha de las diferentes actividades de aprendizaje que iremos presentando a lo largo de este artículo.

Quizás sea un obviedad comentar que el camino de la innovación docente no lo iniciamos ayer; de hecho, ambas pensamos que siempre hemos estado innovando en mayor o menor medida. Pero, hemos resuelto que está bien poner una fecha al período en el que se fue fraguando nuestro trabajo conjunto y decidido en el campo de la innovación docente: marzo de 1999. Pronto cumpliremos 10 años que han estado jalonados de estudio, investigación y puesta en práctica de distintos proyectos. Ya desde esa fecha inicial se fue forjando el grupo de investigación en innovación docente del que formamos parte y que, en los últimos años, denominamos Formación Matemática en Ingeniería (en adelante, FMI), reconocido como tal por la UZ y apoyado en su andadura por el Instituto de Ciencias de la Educación de la UZ.

El nombre del grupo FMI ya insinúa cuál es el ámbito docente de nuestro interés. Estamos adscritas como profesoras del departamento de Matemática Aplicada a uno de los dos centros de la ciudad de Zaragoza que imparten titulaciones de Ingeniería (CPS: Centro Politécnico Superior y EUITIZ: Escuela Universitaria de Ingeniería Técnica Industrial de Zaragoza). En consecuencia, nuestra experiencia docente se ha concentrado en las asignaturas de matemáticas de alguna titulación de Ingeniería.

Hemos coordinado e intervenido en diferentes Proyectos de Innovación subvencionados por la UZ en los que hemos alcanzado diferentes objetivos tales como estudiar y evaluar el perfil de ingreso competencial de los estudiantes que acceden a las titulaciones de Ingeniería (Riaguas, Arribas, Celorrio y Lerís, 2006; Boal y otros, 2007; Bueno y otros, 2008 y Boal, Bueno, Lerís y Sein-Echaluce, 2008-2) o diseñar e impartir cursos de formación para los estudiantes de nuevo ingreso (Boal, Arribas, Leris y SeinEchaluce, 2008-1 y Boal, Lerís y Sein-Echaluce, 2008-3) o crear materiales para la docencia presencial y no presencial o evaluar la calidad de los cursos que teníamos publicados en el ADD.

Durante el trienio 2006-08, el Ministerio de Educación y Ciencia ha financiado el proyecto TSI 2005-04127 "e-LKG Platform: Nueva Plataforma de servicios integrados de Gestión del Conocimiento, Trabajo Cooperativo y e-Learning a partir de software libre. Experimentación y puesta en servicio", en el que hemos participado tres Universidades españolas: Zaragoza, Jaume I de Castellón y Politécnica de Madrid, y en el que se ha unido desarrollo tecnológico y experimentación aplicada a la formación matemática. Hemos construido en colaboración con Consorzio Interuniversitario Lombardo per L'Elaborazione Automatica, abreviadamente CILEA CILEA, un entorno de aprendizaje online que sirve de apoyo a los estudiantes que necesitan las 
matemáticas en las titulaciones universitarias que van a iniciar o ya están iniciando.

Somos conscientes de que el conocimiento, sobre cualquier asunto, está distribuido de forma que nadie lo sabemos todo y entre muchos lo sabemos casi todo. Por eso, creemos que mirar hacia otros grupos de innovación docente es parte de la tarea emprendida. Estamos muy agradecidas al apoyo del Laboratorio de Innovación en Tecnologías de la Información de Universidad Politécnica de Madrid, en particular, a Ángel Fidalgo con el que hemos colaborado en el uso de plataformas para e-learning y para el trabajo cooperativo. Mantenemos una estrecha colaboración con grupos españoles de la Universidad Jaume I de Castellón, de la Universidad Politécnica de Madrid y del Centro de Innovación para la Sociedad de la Información de la Universidad de Las Palmas de Gran Canaria. También podemos decir que formamos parte del entramado del próximo EEES pues colaboramos habitualmente con el Institute of Technology of Sligo de Irlanda, con la Università Cattolica del Sacro Cuore de Milán y Università degli Studi Milano Bicocca de Italia y con la Technical University of Ostrava de la República Checa.

\section{El CONTEXTO: FORTALEZAS Y DEBILIDADES}

En este artículo exponemos algunas de las experiencias e iniciativas docentes que hemos llevado a cabo en las asignaturas de Fundamentos Matemáticos de la Ingeniería de la titulación de Ingeniería Técnica Industrial, especialidad Electricidad y de Álgebra de Ingeniería Informática en la Universidad de Zaragoza.

Ambas asignaturas tienen en común que se estudian en el primer curso de cada uno de los grados mencionado y que, por tanto, reciben estudiantes recién incorporados a sus estudios universitarios. También común a ambas es el elevado número de estudiantes matriculados, alrededor de 80 en la asignatura de Fundamentos Matemáticos de la Ingeniería y en torno a 130 en Álgebra de Ingeniería Informática. Pero también hay diferencias claras. Una de ellas proviene del hecho de que corresponden a estudios conducentes a una titulación de grado medio y otra de grado superior, por lo que eso conlleva respecto a los requisitos legales de acceso y, en consecuencia, a la formación preuniversitaria con la que acceden los estudiantes de cada titulación. Otra diferencia de gran calado docente radica en que la asignatura de Fundamentos Matemáticos de la Ingeniería es una asignatura de duración anual, de 15 créditos docentes, mientras que la asignatura de Álgebra de Ingeniería Informática se encuadra en el primer cuatrimestre con 6 créditos docentes.

El Plan de Ordenación Docente aprobado para ambas asignaturas contempla la realización de sesiones presenciales del grupo completo en el aula tradicional y de sesiones, también presenciales, de trabajo en grupos pequeños en el aula de ordenadores. Se trata de dos asignaturas teóricoprácticas, que pretenden el desarrollo de competencias específicas matemáticas como:

- Conocer y saber usar los contenidos matemáticos descritos en el Plan de Estudios.

- Potenciar y/o mejorar el uso de lenguaje matemático y simbólico.

- Desarrollar las formas de pensar, razonar y argumentar.

- Mejorar y diversificar las formas de representar y comunicar información y resultados de carácter científicomatemático.

- Fomentar y capacitar en el uso de las nuevas tecnologías para la resolución de modelos matemáticos procedentes de diferentes contextos.

Y de otras competencias genéricas como:

- Mejorar las habilidades de comunicación tanto oral como escrita.

- Promover e incentivar el diálogo y el trabajo en grupo.

- Desarrollar la capacidad de ser un aprendiz autónomo, en particular, de matemáticas.

Es indudable que el diseño de un curso en el que se pretende realizar algunos cambios, entre ellos usar una plataforma de e-learning, ha de estar condicionado a un análisis de las fortalezas y debilidades del contexto en el que se pretenden aplicar. El plan de acción y, en definitiva, el proyecto se verá afectado por las circunstancias de cada situación.

A continuación exponemos algunos datos y peculiaridades de nuestro contexto de aplicación, al menos los que hemos 
considerado más relevantes para tomar algunas decisiones sobre los recursos de Moodle a utilizar y la forma e intensidad de su utilización. Algunos datos se refieren a dos estudios del perfil de ingreso del estudiante de Ingeniería, uno en septiembre de 2004 (Riaguas, Arribas, Celorrio y Leris, 2006) y otro en septiembre de 2006 (Bueno y otros, 2008). Aunque ahora nos interesan los datos más cercanos de septiembre de 2006, haremos mención de la evaluación precursora de 2004 cuando interese mostrar la evolución del alumnado como desencadenante de nuevas actividades de aprendizaje.

- La habilidad del estudiante en el uso de las nuevas tecnologías es hoy día una fortaleza.

Una de las competencias que es mencionada en la introducción de las asignaturas de matemáticas del Bachille- rato LOGSE se refiere al uso de los recursos tecnológicos; competencia que es concretada al objetivo 8 de la LOGSE que dice: "Servirse de los medios tecnológicos que se encuentran a su disposición, haciendo un uso racional de ellos y descubriendo las enormes posibilidades que nos ofrecen."

En las dos evaluaciones del perfil de ingreso, pedimos al estudiante que se calificara a sí mismo como usuario de algunos recursos tecnológicos. En particular, en la evaluación de septiembre de 2006 se les preguntó sobre su habilidad en el uso de la calculadora, del correo electrónico o Messenger, de la red internet, de programas ofimáticos como word o excel y de programas científicos como Derive o Mathematica. Obtuvimos los resultados que se ven en el gráfico 1.

Gráfico 1. Habilidad en el uso de nuevas tecnologías.

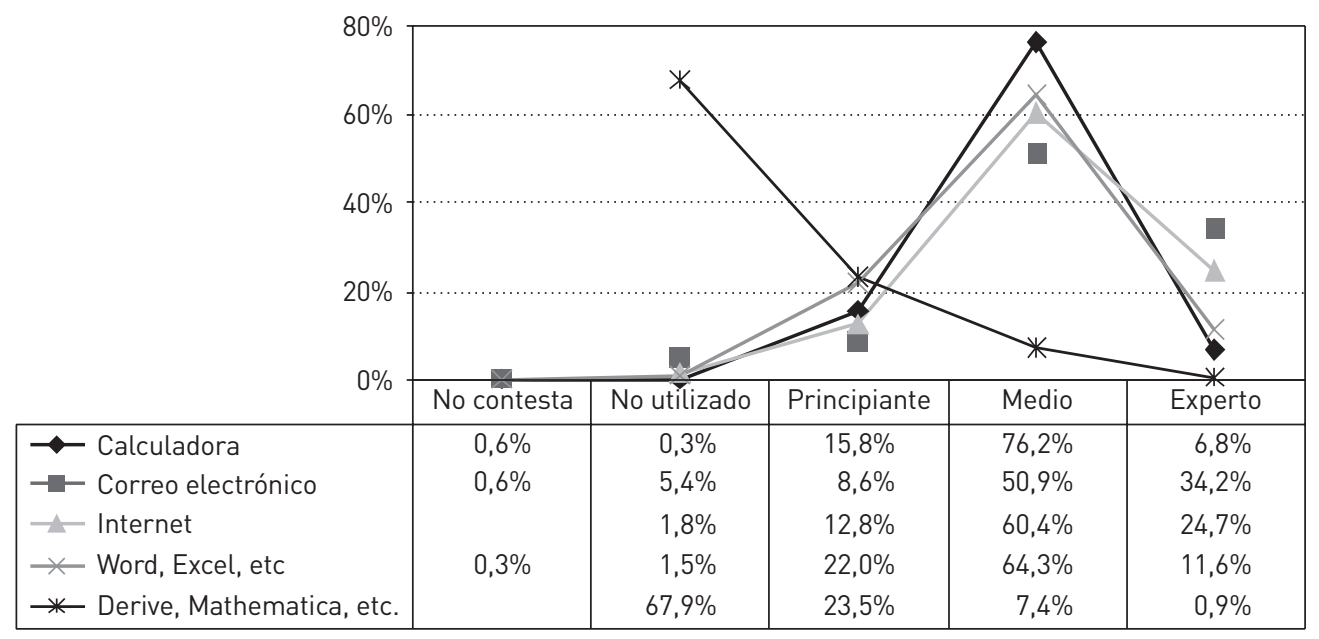

La evolución del alumnado en este asunto ha sido extraordinaria, pues de un $12 \%$ de estudiantes que declaraba navegar por Internet en septiembre de 2004, hemos pasado a que un $85,1 \%$ de los jóvenes que acceden a la Universidad se declaren usuarios de nivel medio o alto de Internet y de sus herramientas de comunicación.

Estos excelentes datos afianzaron la idea de dar un nuevo impulso al uso de las nuevas tecnologías en el diseño del aprendizaje de nuestras asignaturas.
- La autonomía del estudiante para gestionar su aprendizaje es una debilidad que ha de tenerse en cuenta en el plan de acción.

Hemos de reconocer que los profesores universitarios exigimos de nuestros estudiantes que se responsabilicen de su aprendizaje, lo que, en muchos casos, significa que pedimos que sean capaces de gestionar autónomamente su tarea de aprender. En principio, tres aspectos nos parecen claves para que un aprendiz pueda realizar su trabajo con 
autonomía: la confianza o seguridad como usuario de las matemáticas, las fuentes de información que suele utilizar y la organización de su tiempo de estudio.

La seguridad en su trabajo y en sus resultados es un valor imprescindible para que el aprendiz no se sienta abru- mado ante las tareas que se le proponen. Los datos que obtuvimos, véase el gráfico 2, nos dicen que poco más del $36 \%$ de los recién ingresados en los estudios de Ingeniería tienen seguridad de la validez o veracidad de las respuestas que ellos producen en la mayoría de los ejercicios de matemáticas.

Gráfico 2. Seguridad en el resultado al terminar un problema.

Cuando has terminado un ejercicio o problema de Matemáticas,
¿sueles estar seguro del resultado obtenido?
Casi siempre
Algunas veces
Casi nunca
$\square$,

En otra pregunta se les sugirieron diferentes formas de conseguir información sobre matemáticas, desde preguntar al profesor, a los compañeros o a los padres hasta la búsqueda de información en libros, en Internet o en otros. Los estudiantes de primer curso de Ingeniería muestran su predilección por preguntar a las personas de su entorno para obtener información de matemáticas, muy pocos, 17,5\%, acuden a los libros y algo menos del 5\% han buscado en la red. Parecen mostrar cierta dependencia de que sea otra persona la que les descifre las matemáticas escritas en un libro o en internet. Estos datos nos revelan la necesidad de que las actividades de aprendizaje virtuales vayan acompañadas de un contrapeso presencial, en tanto el estudiante no vaya acostumbrándose al nuevo entorno de aprendizaje.

Gráfico 3. Forma de resolver dudas: búsqueda de información.

Si alguna vez tienes dudas o no comprendes "algo" de las matemáticas. ¿Cómo las resuelves?

Pregunto a mis compañeros Pregunto a mi profesor o profesora Pregunto a mis padres

Voy a una academia

Consulto libros

Conecto con chats o foros sobre el asunto Busco información en internet

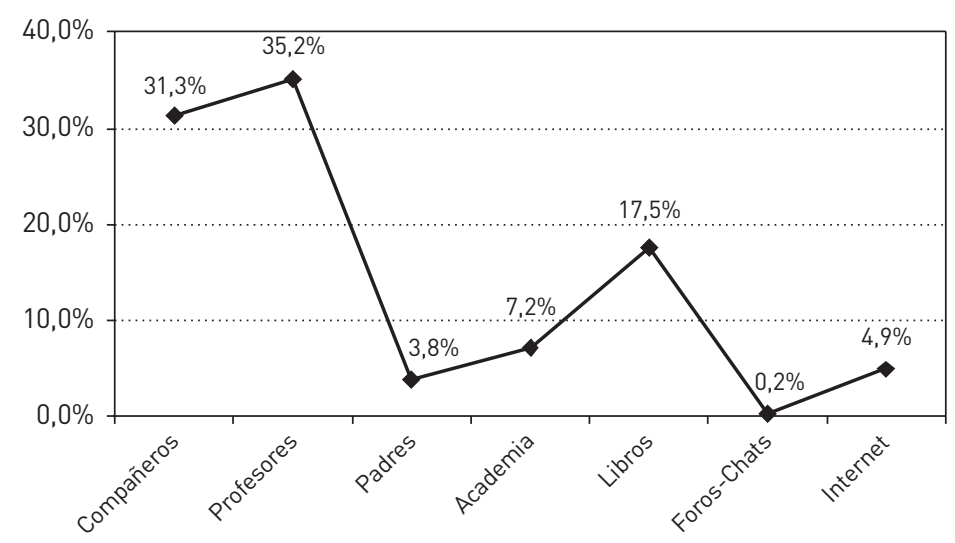


Finalmente, otro aspecto importante es saber su capacidad para organizar su tiempo de estudio. Merece nuestra atención, véase el gráfico 4, que casi un $33 \%$ de los estudiantes sólo estudia matemáticas antes de los exámenes, es decir, sólo responde ante el estímulo del examen o, quizás, ante el estímulo de la valoración de su aprendizaje: esto es un signo de las dificultades del estudiante para programar personalmente su estudio. Es necesario tener en cuenta esta realidad en el diseño de la enseñanza.

Gráfico 4. Asiduidad en el estudio de matemáticas. ¿Con qué asiduidad has estudiado las Matemáticas durante
un curso escolar?

Todos o casi todos los días Un día a la semana

Sólo antes de los exámenes

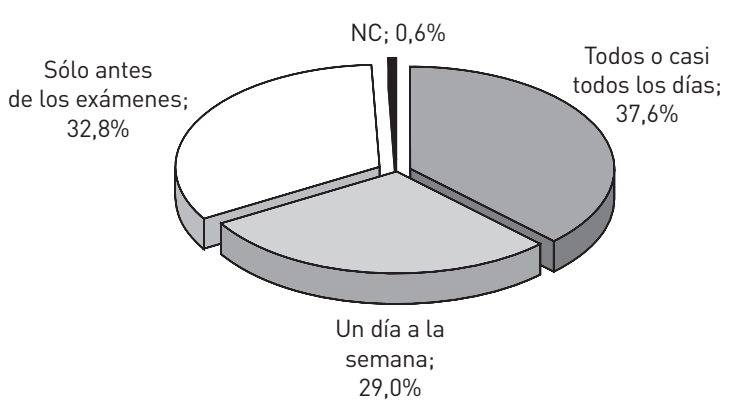

\section{- Los recursos tecnológicos disponibles han crecido de tal manera que es una oportunidad que hemos de aprovechar}

Al pretender que algunas actividades de la enseñanza se realizaran utilizando las herramientas que las plataformas de e-learning ponen a nuestra disposición, es imprescindible conocer hasta qué punto los estudiantes de esa enseñanza disponen de los recursos tecnológicos necesarios. Hay que tener en cuenta dos datos extraordinariamente positivos y otro que podría suponer una amenaza.

Los resultados de la encuesta sobre equipamiento y uso de tecnologías de la información y comunicación en los hogares, publicados en la web del Instituto Nacional de Estadística www.ine.es, muestran una evolución ascendente en cuanto al porcentaje de viviendas de Aragón que disponen de ordenador personal (ordenador de sobremesa, ordenador portátil) y al porcentaje de ellas que disponen de acceso a Internet. Otro dato muy interesante es la relación de recursos materiales de los Centros Universitarios, en especial, la dotación de ordenadores de las salas de usuarios y su disponibilidad y suficiencia para garantizar el acceso de los estudiantes a los recursos y actividades de aprendizaje via web. En el caso del campus Rio Ebro de la UZ, la puesta en marcha en 2006 de unos 70 ordenadores conectados a la red en la sala de estudio de uno de los edificios supuso un salto cualitativo importante de cara a garantizar la accesibilidad al entorno de aprendizaje virtual puesto en marcha.

Un hecho a considerar: los estudiantes desplazados del domicilio familiar. Los estudiantes que tienen que desplazarse desde el lugar de residencia familiar hasta la ciudad donde inician sus estudios universitarios no dispondrán de conexión a Internet en el lugar de alojamiento salvo, quizás, en el caso de que estén instalados en Colegios o Residencias Universitarias.

\section{- La actitud pasiva del estudiante ante su propio aprendizaje es una amenaza para el éxito de las prácticas docentes más activas.}

El modelo de enseñanza predominante en la educación española propicia la pasividad del estudiante ante el aprendizaje. Hasta tal punto está enraizada la actitud pasiva que no es extraño que los estudiantes demanden al profesor la repetición de ese modelo en el que saben cómo actuar y con el que, en su mayoria, han tenido éxito. No obstante, esta tensión es una resistencia al cambio, resistencia que suele superarse al ver cómo son valorados positivamente sus nuevos logros. 
- Una nueva etapa educativa es un momento de cambio y es una oportunidad para la innovación.

Al contrario de lo dicho en el punto anterior y a pesar de algunos lastres, el iniciar una nueva etapa educativa hace que el estudiante sea receptivo al uso de nuevas herramientas de aprendizaje e incluso esté dispuesto a probar nuevas metodologías. A nuestro juicio, la introducción progresiva $y_{1}$ a la vez, constante de las nuevas tecnologías y metodologías es una de las claves para paliar la ansiedad de lo desconocido y animar a la participación.

\section{- La inestabilidad del grupo docente en los primeros meses del curso reduce el tiempo en que pueden aplicarse determinados cambios docentes.}

En nuestro caso, los estudiantes pueden cambiar el grupo de docencia asignado en la matrícula e incluso matricularse hasta bien entrado el mes de noviembre, lo cual genera inestabilidad en el grupo docente y es, desde luego, una amenaza 0 , más bien, un impedimento para la puesta en marcha de algunas prácticas educativas innovadoras como el trabajo en grupos formales o estables.

La escasa o nula experiencia de trabajo en pequeños grupos, salvo en actividades deportivas, es una debilidad con la que hemos de contar al proponer tareas cooperativas en grupos formales o informales.
El trabajo en equipo es una competencia transversal que ha de desarrollarse en la etapa universitaria sobre la base de cierta experiencia en etapas educativas anteriores. En nuestra docencia proponemos a los estudiantes unos pocos trabajos en equipo, con la idea de promover su capacidad de colaboración, de contrastar ideas, de saber argumentar, de tomar decisiones.

En la evaluación de septiembre de 2006, preguntamos directamente al estudiante sobre su experiencia en el trabajo en equipo y obtuvimos los resultados que se muestran en el gráfico 5 . En ellos se observa que en torno a un $57 \%$ de los encuestados manifiesta una nula o escasa experiencia en el trabajo en grupo. No es de extrañar pues que los grupos de trabajo en este nivel adopten estrategias de trabajo consistentes en repartirse la tarea entre los miembros del grupo sin que haya interrelación entre unos y otros. La consecuencia es que el trabajo en pequeños grupos ha de estar organizado y guiado por la profesora de modo que se aprendan y adopten las verdaderas estrategias de un buen trabajo en equipo.

Las fortalezas y debilidades mencionadas son las que hemos detectado en nuestro entorno docente que a nuestro juicio han tenido y tienen incidencia en muchas decisiones sobre el diseño de las actividades que proponemos a nuestros estudiantes. Quizás algunas de ellas sean extrapolables a otras situaciones docentes.

Gráfico 5. Trabajos o proyectos en grupo.

Recuerda tus estudios previos: ¿cuántos trabajos o proyectos en grupo has realizado?

Muchos (más de 8)

Bastantes (entre 4 y 8 )

Pocos (entre 1 y 3 )

Ninguno $\square$,

$\square$,

$\square$,

$\square$

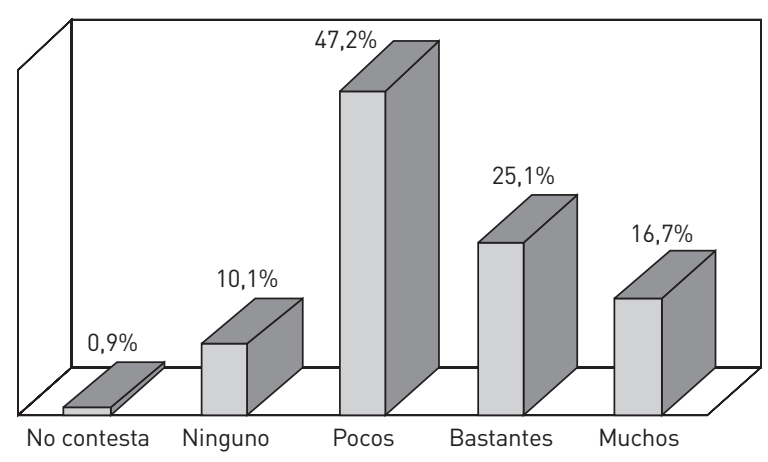




\section{Nuestras asignaturas}

La pequeña historia de nuestras aventuras en el uso de herramientas de e-learning y de nuevas metodologías en el aula no sigue la secuencia con la que vamos a describir qué, para qué y cómo usamos diferentes actividades de aprendizaje.

No hay que olvidar que las asignaturas de las que somos responsables pertenecen al ámbito de la enseñanza presencial de la Universidad de Zaragoza y, por tanto, no es necesario resolver ninguno de los problemas ligados al modelo de formación a distancia mediante el uso de medios electrónicos. Más bien al contrario, son las herramientas electrónicas las que han de echar una mano.

Hermann Ebbinghalls establece que durante el proceso de aprendizaje somos capaces de retener un $10 \%$ de lo que leemos, $20 \%$ de lo que oímos, $30 \%$ de lo que vemos, $50 \%$ de lo que vemos y oímos, $70 \%$ de lo que decimos y $90 \%$ de lo que decimos y hacemos. Otras ideas sobre cómo hacer interactivo el aprendizaje se pueden encontrar en Bergeron, 2006; Instructional Strategies Online, 2006.
El objetivo de esta experiencia es incorporar en nuestra docencia, de una forma progresiva, actividades que requieran la participación activa del estudiante $y$, de ese modo, sean ellos los protagonistas de su propio aprendizaje. Atendiendo a la clasificación de Ebbinghalls, pretendemos que nuestros estudiantes se sitúen en los niveles de retención máximo proponiéndoles que "hagan" y "digan".

Necesitamos hacer una pequeña incursión en el área de las matemáticas para explicar cómo abordamos el proyecto de innovar para mejorar el aprendizaje de nuestros estudiantes. Todo parte de nuestra concepción de la materia, las matemáticas, que el estudiante debe llegar a comprender. Advirtamos que se trata de nuestras reflexiones particulares y que están sujetas a toda suerte de modificaciones y mejoras; pero hemos de tomar un punto de partida y este es el nuestro. Entendemos que un concepto matemático engloba desde la idea o definición del concepto hasta las estrategias de su uso, pasando por toda clase de propiedades. El esquema podría ser algo asi:

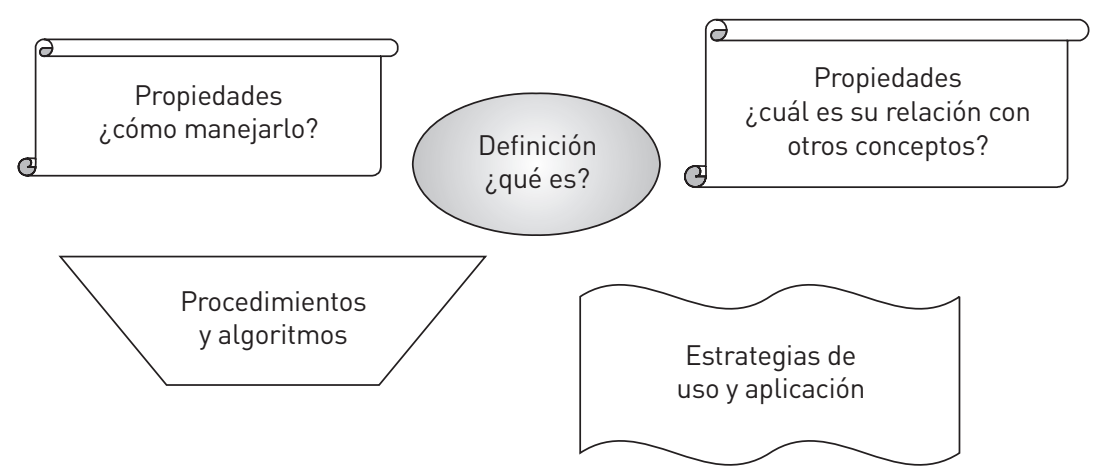

Concepto matemático.

El objetivo del estudiante es comprender un concepto matemático. Para ello es necesario que el estudiante conozca, se entrene y reflexione sobre todos los aspectos que integran ese concepto. Por tanto, es importante que disponga de información suficiente, que se le faciliten medios de entrenamiento adecuados y que se organicen actividades que propicien un aprendizaje profundo.
Ponemos a disposición del estudiante recursos estáticos, diseñamos actividades para el trabajo individual y organizamos actividades para el trabajo en grupo en el ADD. Todas ellas están integradas en el proyecto de enseñanzaaprendizaje de cada una de las asignaturas y entrelazadas con el trabajo que se desarrolla en las clases presenciales. 


\section{El diseÑo de LOS CURSOS EN EL ADD}

Hemos de reconocer que en los momentos iniciales de la andadura de estos cursos parecía que había que "engancharse al e-learning" como fuera, pero la experiencia de estos años han puesto las "cosas en su sitio". Sólo unas reflexiones, fruto de la experiencia:

- El aprendizaje apoyado por el ordenador no es un objetivo sino un planteamiento de la enseñanza para utilizar otros recursos que nos permitan alcanzar los auténticos objetivos.

- No es obligatorio que el curso use cuantas herramientas tiene Moodle. No todas las asignaturas necesitan un chat, un foro, páginas de presentación, un taller, etc., no pretendamos utilizar unos determinados recursos y además en todos los procesos formativos.

- Es importante recordar que las asignaturas de cuya docencia somos responsables disponen de la gran ventaja de la presencialidad $y_{1}$ en consecuencia, de la riqueza de la comunicación cara a cara entre los actores del proceso de enseñanza-aprendizaje.

Por tanto, nuestro planteamiento del curso en el ADD es el de un complemento más dentro del proyecto de aprendizaje de cada una de las asignaturas y como tal ofrece herramientas $y$, por tanto, posibilidades metodológicas que se agregan a la docencia presencial. El diseño metodológico y la organización de los recursos responden a esa idea de complementariedad.

En nuestra opinión el trabajo del estudiante debe reconocerse en los términos que a él le interesan: una mejora en la calificación final. Y así lo hacemos. En ambas asignaturas hemos ido ofreciendo una mejora en la calificación final en función del valor de la actividad realizada por cada estudiante; la cuantía de esa mejora depende de la diferente programación de actividades en cada curso y asignatura. Por ejemplo, el valor de un buen trabajo continuo durante el actual curso académico 2008-09 es del 20\% de la calificación final.

De las formas que ofrece Moodle para organizar los recursos, hemos elegido la que distribuye la hoja principal en secciones o temas, término este último que trataremos de evitar para no confundir al lector con la idea de un tema de los contenidos de la asignatura. Cada sección, salvo dos, está dedicada a una unidad de aprendizaje (formada por los contenidos de la asignatura que pueden estar en uno o varios temas) en la que se ubican todos los recursos y actividades relativos a ella. Hemos procurado que el entorno virtual, su aspecto, fuera claro y sencillo. En todas las secciones o unidades de aprendizaje hemos mantenido una estructura común con el objetivo de facilitar la localización de los distintos tipos de recursos.

Además de las secciones dedicadas especificamente al aprendizaje hemos incluido dos o tres más, según la asignatura, con contenidos y estructura diferente. Una de ellas, la primera que aparece en la pantalla inicial del curso, está dedicada a ofrecer al estudiante un punto más de información general sobre la asignatura y de comunicación de carácter general.

Como se ve en la figura 1, todos los datos e informaciones de la asignatura que deben ser conocidos por los usuarios de la misma y que, por ello, se publican en los tablones del Centro y del Departamento encuentran un hueco en esta sección inicial:

- La información sobre la asignatura se sitúa en la parte superior. El primer enlace nos lleva a una página web con la información del curso, se trata de una versión ampliada de la hoja informativa que se reparte a los estudiantes durante las primeras clases presenciales, y los dos siguientes al horario de las tutorías académicas presenciales.

- Bajo el título de tablón de anuncios, está ubicada la información sobre los exámenes: las fechas previstas para la realización de todos los exámenes de primer curso de la titulación de la asignatura en cuestión, la convocatoria del siguiente examen de la asignatura y las notas de exámenes anteriores.

- Dos foros de comunicación también general: novedades y cafetería. El foro de novedades es usado esencialmente para comunicación unidireccional, de la profesora a los estudiantes, para que reciban los mensajes a su correo electrónico con los avisos de carácter general sobre incidencias, modificaciones, notificaciones de interés general. El foro cafetería dispuesto en una de las asignaturas para uso de los estudiantes de modo que dispongan de un lugar para sus propios debates sobre los asuntos que deseen o para incluir información sobre los asuntos que les interesen, aunque no estén directamente relacionados con la asignatura, o para consultar sobre las dificultades que les puedan surgir en el uso 


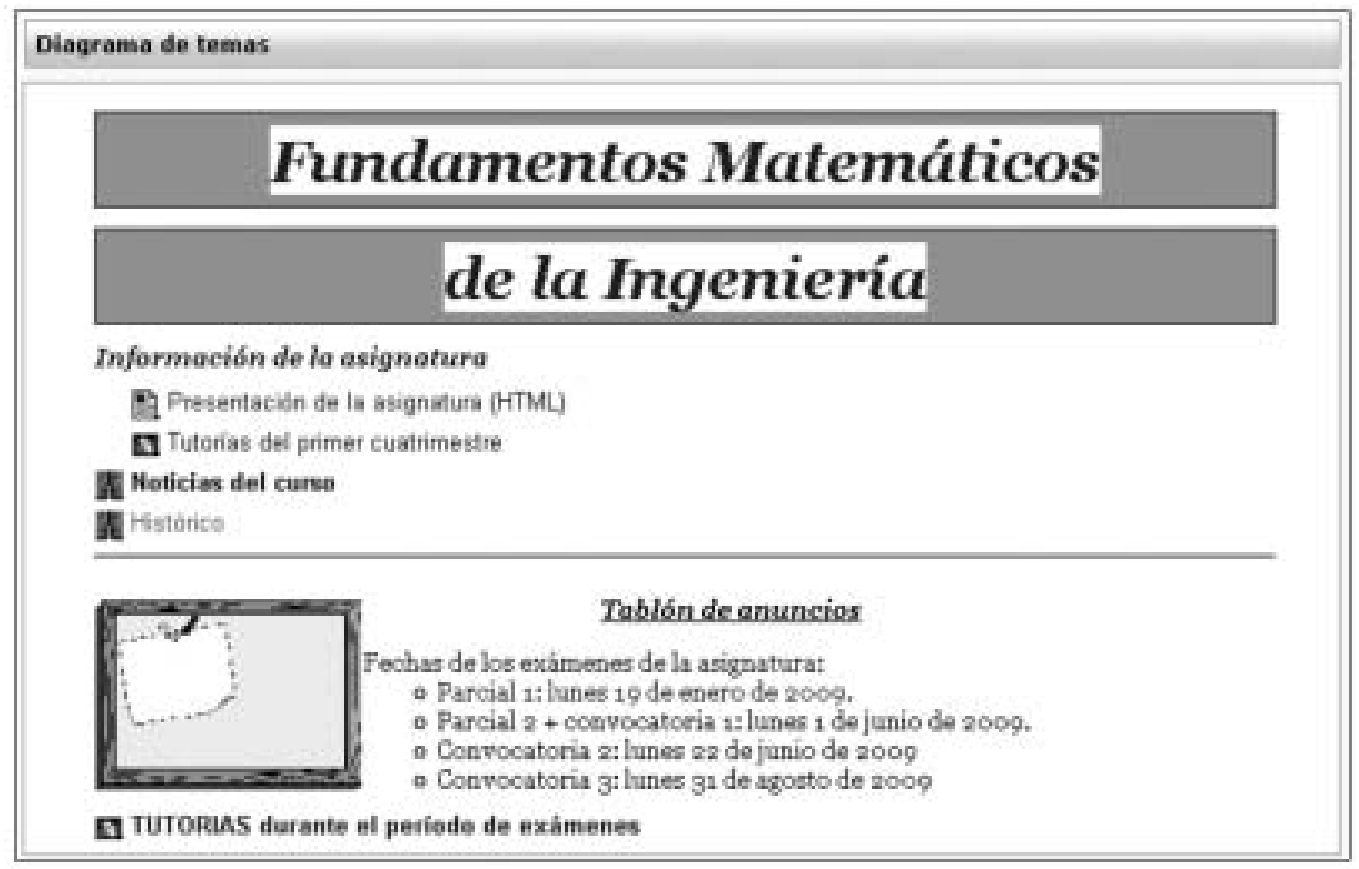

Figura 1. Sección inicial de uno de los cursos.

de la plataforma 0 , incluso, para algunas actividades de entretenimiento y distensión.

Hemos incluido una sección complementaria para recoger en ella otro tipo de información y recursos de interés, por ejemplo, se han publicado los enunciados de los exámenes de cursos académicos anteriores junto a la resolución de algunos ejercicios de examen realizados por los estudiantes, digamos que es el lugar de recursos compartidos que pueden estar generados por los profesores o por los estudiantes.

En ambas asignaturas, las prácticas con ayuda de programas científicos (MatLab o Mathematica) en grupos reducidos tienen un peso importante, el 20 ó $25 \%$. Una sección más de Moodle reúne los recursos, información y actividades especificamente dedicadas a esa parte de la asignatura.

\section{LAS UNIDADES DE APRENDIZAJE: METODOLOGÍA Y ACTIVIDADES}

Nuestra experiencia de aprendizaje se apoya esencialmente en el método conductista con incursiones en técnicas constructivistas. La metodología de trabajo es una mezcla entre el trabajo individual y el trabajo en grupo, pero en cualquier caso, fomentando la participación activa de los estudiantes.

El modelo o la estructura de las unidades de aprendizaje responde al siguiente esquema: repositorio de información de los tópicos matemáticos, cuestionarios de aprendizaje, tareas o problemas y encuestas de reflexión. Todas las unidades contienen su propio repositorio de información y uno o varios cuestionarios sobre los tópicos que se abordan en la unidad. La presencia de tareas o de encuestas no es una regla común a todas las unidades, aunque siempre hay propuesta alguna de estas actividades.

\section{- Sobre el repositorio de información de los tópicos matemáticos}

Cada unidad contiene información y documentación sobre el o los conceptos matemáticos que la conforman. Los materiales de teoría, de problemas y de prácticas se presentaron de forma escalonada en el tiempo, de acuerdo con las clases presenciales. 
En cada tema de estas unidades se accede a archivos creados específicamente para el entorno web. En cada uno hemos tratado de

- Sintetizar la información siguiendo un esquema común y procurando que no fueran muy extensos.

- Describir, en forma de guión, cuáles son las claves o los puntos importantes de cada tema.

- Recordar cuáles son las ideas o procedimientos que es necesario conocer previamente y dónde pueden consultarse, si se necesita.

- Incluir el listado de ejercicios y problemas de ese tema.

- Recordar unos pocos libros de consulta adaptados al nivel del curso.

- Mostrar algunas órdenes de trabajo que el programa Mathematica ofrece.

Además, en algunos de esos temas hemos aprovechado los materiales que existen en Internet y se hacen recomendaciones de que accedan a determinadas direcciones web para completar información sobre aspectos matemáticos relevantes o para conocer hechos históricos, curiosidades o incluso pasatiempos. Con la inclusión de referencias de otras páginas web publicadas en Internet se obtienen otros beneficios además de los directamente relacionados con el aprendizaje de la materia. Se trata de que:

-El estudiante se acostumbra a leer información en la red. Además observa una buena conducta, la de indicar siempre de dónde procede o de quién es la autoría de la información que se ha recogido.

-El estudiante dispone de modelos de páginas web de calidad y así puede desarrollar criterios para valorar la información que localice por la red $y$, por tanto, generar sus propios indicadores de calidad.

- El estudiante descubre, si no lo sabía ya, la necesidad de aprender inglés.

Esto nos lleva a comentar otra de las iniciativas llevadas a cabo y que consiste en la inclusión de un tema en un foro general en el que se sugiere a los estudiantes que localicen y recomienden alguna página web que sea interesante para la asignatura. El tipo de enlaces que se pueden incluir son de naturaleza muy diversa, por ejemplo, páginas con material docente, programas, aplicaciones, curiosidades matemáticas,... Entre los objetivos que se consiguen al construir esta agenda no sólo está el hecho de construir una lista de enlaces "útiles", sino lo que es si cabe más importante, que los estudiantes busquen información, la clasifiquen como relevante y emitan una opinión sobre la página. Han pasado de ver lo que la profesora elige a tener que formarse un criterio propio para elegir.

En resumen, el repositorio de información está formado primordialmente por archivos generados y publicados por la profesora (información estática publicada en función del calendario docente) con enlaces, en algunos casos, a páginas web externas y además las aportaciones de los estudiantes, información cambiante o más dinámica, sobre las páginas web que ellos recomiendan.

\section{- Sobre los cuestionarios de aprendizaje}

En cada tema se proponen uno o varios cuestionarios de aprendizaje. Reiteramos que se trata de una actividad totalmente engarzada en el proyecto de aprendizaje $y$, por tanto, la configuración de los cuestionarios depende de los conceptos que traten y del calendario de trabajo. Durante estos tres últimos cursos académicos nos hemos dedicado a crear preguntas de opción múltiple, la mayoría, o de emparejamiento o de respuesta abierta corta de forma que estamos llegando a construir un pequeño banco de preguntas.

Hemos puesto en práctica dos tipos de configuración de los cuestionarios; en un caso las preguntas del cuestionario son fijas y en otro se realiza una selección aleatoria dentro de las categorías que contienen preguntas de características análogas. En ambos casos, se ofrece al estudiante la posibilidad de realizar dos intentos por cuestionario y así se permite al estudiante enfrentarse, en el segundo intento, a las mismas preguntas (de las que no se le proporciona las soluciones) $u$ otras nuevas, análogas a las del primero, con el conocimiento adquirido en el primer intento. La mejoría en los resultados suele ser el denominador común, lo cual, en nuestra opinión confirma que la actividad de responder a cuestionarios produce aprendizaje.

En el párrafo anterior hemos afirmado que el estudiante adquiere conocimiento como consecuencia de realizar el primer intento del cuestionario. Es evidente que al tratar de responder a las preguntas el estudiante está aprendiendo, pero hemos de decir que las respuestas del estudiante están acompañadas de retroalimentación de dos tipos: $a$ 
priori y a posteriori. Con retroalimentación a priori nos referimos a la que forma parte de la pregunta y que la hemos incluido para que aparezca una vez el estudiante haya respondido al cuestionario. En algunas preguntas hay retroalimentación general (véase la figura 2), es decir, comentarios e información que ayuda al estudiante a di- rigir su estrategia para responder a ese tipo de preguntas. $Y$ en muchas otras hay retroalimentación especial para los distractores (véase la figura 3) que en la pregunta de opción múltiple se ofrecen, estos distractores suelen reflejar errores comunes advertidos a lo largo de nuestra experiencia docente.

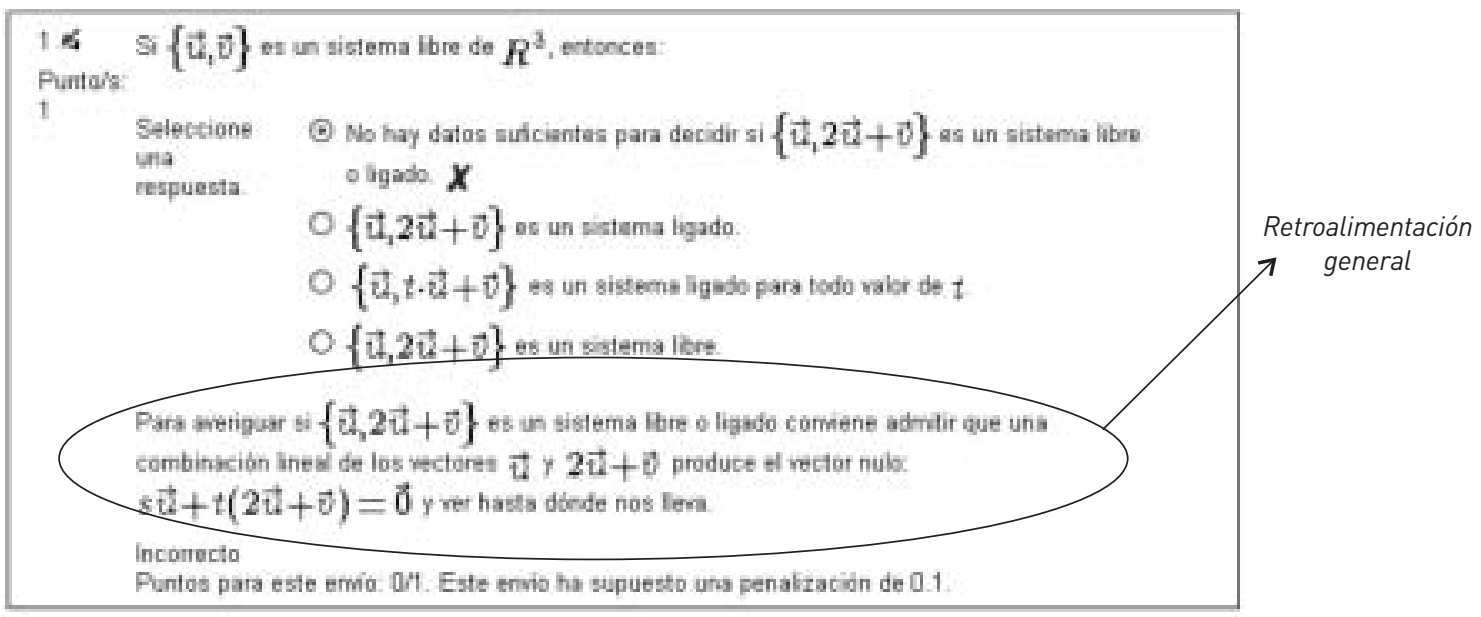

Figura 2. Retroalimentación general en una pregunta.

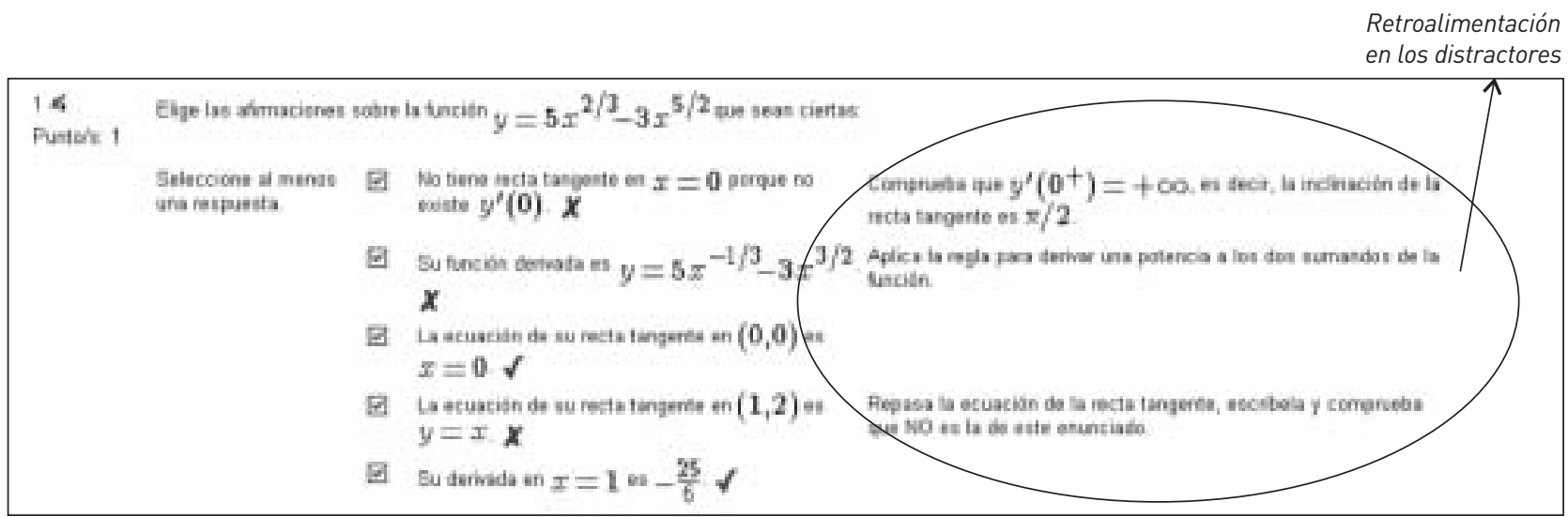

Figura 3. Retroalimentación en cada uno de los distractores de una pregunta. 
Ahora bien, además de lo previsto a priori, conviene reaccionar ante lo observado y dar retroalimentación a posteriori. Así pues, es recomendable consultar la estadística de respuestas a cada opción de las preguntas de los cuestionarios y decidir si es necesaria una intervención especial o una retroalimentación a posteriori. Nosotras hemos usado dos técnicas: comentar los errores o dificultades en la clase presencial y/o abrir un foro en el que abrimos uno o varios temas de modo que se oriente el debate hacia la aclaración del error o errores o hacia la explicación de la solución.

Otro asunto ha merecido nuestra atención a lo largo de estos dos cursos de implantación de la actividad de cuestionarios: el periodo de tiempo en el que parece conveniente que estén disponibles para el estudiante. La metodología que hemos comentado lleva implícito que los cuestionarios estén disponibles durante un tiempo limitado, pero la experiencia nos ha enseñado que hemos de tener en cuenta que plazos de tiempo muy cortos, tres o cuatro dias, pueden hacer imposible la participación de los estudiantes (dificultades de calendario, de conexión a internet, etc.). Asi que, este curso académico, estamos dejando abiertos los cuestionarios entre 7 y 10 días tras la presentación de la correspondiente materia en la clase presencial. A ello hemos de añadir que los cuestionarios se vuelven a abrir para la preparación de exámenes durante los períodos del curso sin docencia presencial.

Es evidente que las autoras hemos realizado un notable esfuerzo para producir las preguntas que pueden formar parte de los cuestionarios de aprendizaje, pero ahora el esfuerzo ha pasado al servidor de la plataforma Moodle. Es muy alentador ver en marcha una actividad que, hoy por hoy, no podemos llevar a cabo en su versión presencial: por un lado, el alumno trabaja para responder a unas preguntas, envía sus respuestas al sistema que, a continuación, le provee de retroalimentación y de las soluciones $y$, por otro, nosotras disponemos de retroalimentación sobre cada estudiante y sobre cada pregunta y así poder actuar en consecuencia.

Además, el estudiante va adquiriendo el hábito de organizar su tiempo de aprendizaje distribuyéndolo a lo largo de todo el curso académico y dejando de concentrarlo exclusivamente en las fechas previas a las convocatorias de exámenes.

\section{- Sobre las tareas o problemas}

Las actividades de aprendizaje que agrupamos bajo el nombre de tareas o problemas pretenden construir un nivel más avanzado de conocimiento de modo que el estudiante utilice los conceptos matemáticos aprendidos para resolver problemas en contextos diferentes al puramente matemático. El propósito de las tareas es que el estudiante descubra las estrategias que realmente funcionan para resolver un problema aplicado. Algunos estudiantes quizás ya hayan adquirido algunas habilidades necesarias para abordar con éxito un problema aplicado; en cualquier caso, el objetivo de las tareas es que el estudiante vea las debilidades y fortalezas de las estrategias que él mismo aplica, si las tiene, y las que emplean sus compañeros.

La metodología usada en las tareas es también diversa: trabajo individual y trabajo en grupos informales, en unos casos, y formales, en otros y en cualquier caso se usan las herramientas de Moodle para asistir el trabajo del estudiante, del grupo y de la profesora. La programación de las tareas a lo largo del curso está influida por una de las debilidades que ya hemos señalado: la inestabilidad de los componentes del grupo docente al inicio del curso académico. Por eso, en el primer cuatrimestre se proponen dos tareas para realizar en grupos informales, es decir, en grupos de estudiantes que se crean espontáneamente y exclusivamente para la realización de cada una de esas tareas; mientras que en el segundo cuatrimestre se proponen tres tareas para realizar en grupos formalmente constituidos para su trabajo continuado durante ese período del curso. Junto a este trabajo cooperativo, se programan dos tareas individuales una para cada cuatrimestre del curso.

Al proponer tareas individuales, tratamos de conseguir que el estudiante "haga" y "vea qué hace", es decir, ponemos a prueba su habilidad para generar y usar estrategias de modelización y resolución. Se propone a cada estudiante un problema/trabajo por cuatrimestre, problema que se sitúa en un contexto "cercano" a la realidad y en el que se han de utilizar los contenidos y métodos matemáticos adquiridos durante una parte del curso, así como el uso del "software" manejado en las clases de prácticas. A cada estudiante se le asigna aleatoriamente el problema que le toca resolver de la lista de diez propuestos. Aunque el trabajo está inicialmente planteado de forma individual y la forma de asignación aleatoria pretende garantizar la 
individualidad del trabajo, no planteamos ninguna objeción a su resolución con otros compañeros que hacen el mismo ejercicio. Realmente la situación que se suele dar, en general, es que unos cuantos estudiantes (pocos, en torno al 10\%) deciden resolver juntos el problema común que han de resolver $y$, en este caso, mayoritariamente se agrupan por parejas.

Dos aspectos son claves en la resolución de los problemas: la capacidad de expresión y el uso de los conocimientos adquiridos; por eso, el estudiante es requerido para que explique las ideas, razonamientos, etc. utilizados para resolver el problema en la forma que lo presenta. La valoración de la tarea realizada se basa tanto en criterios de contenidos como en criterios de habilidades. Lo cierto es que la corrección de estas pruebas es una tarea exigente, pues de la revisión de cada trabajo resulta un documento que se envía a cada estudiante en el que les indicamos dónde hay errores de concepto o de método, dónde hay comentarios o expresiones incorrectas, etcétera y cómo pueden subsanarlos autónomamente a través de apuntes, libros, información en el $A D D$ o, en algunas ocasiones, convocamos una tutoría compartida con algunos o todos los compañeros que han hecho la misma tarea.

Respecto a las tareas que se realizan en pequeños grupos de cuatro estudiantes, tanto en el caso de grupos esporádicos como en el de grupos estables, a los objetivos de aprendizaje de las tareas individuales se añaden el de mejorar las habilidades para el trabajo en grupo y desarrollar las capacidades de comunicación oral o escrita. Al trabajar en grupo, todos sus componentes "ven lo que otros hacen" para resolver un problema, es decir, descubren cómo trabajan otros compañeros y qué estrategias utilizan.

La metodología de trabajo en las tareas en pequeños grupos combina el uso de herramientas de Moodle con actividades presenciales. En cada tarea, el grupo mantiene una 0 dos reuniones con la profesora en las que el grupo expone el estado del trabajo realizado y las dificultades encontradas, resueltas o no; nuestro papel consiste en orientar, no resolver, el trabajo del grupo a través de sugerencias de estudio 0 a través de preguntas que conduzcan hacia estrategias más productivas 0 , en definitiva, a través de cualquiera de las múltiples iniciativas que tomamos los profesores ante las dificultades o dudas en el aprendizaje de nuestro estudiantes. El grupo dispone de un foro particular en Moodle del que también forma parte la profesora, nuestras intervenciones en este foro se reducen a aquellos momentos en los que observemos errores o dificultades que no se superan o en caso de inactividad del grupo. El resultado del trabajo debe reflejarse en un documento escrito, que en unos casos es expuesto públicamente en la clase presencial y en otros publicado en Moodle.

Una iniciativa reciente es la de adjuntar una rúbrica a las tareas programadas, es decir, una guía de trabajo que se entrega a los alumnos antes de iniciar una determinada tarea para ayudarles a pensar sobre los criterios con los que su trabajo será juzgado. Esperamos que esta decisión redunde en una mejora de los resultados de aprendizaje.

\section{- Sobre las encuestas de reflexión}

Existen numerosas técnicas de dinámicas de grupo que promueven la participación de los estudiantes en las clases magistrales. Hemos ensayado en las clases expositivas dos técnicas: "Think-Pair-Share" (Creed,1996), método en el que se hace partícipe a los alumnos de lo que se está explicando proponiendo cuestiones sencillas sobre las que debaten los estudiantes por parejas y "One minute paper" (Ross y Angelo, 2001 y Stead, 2005) en el que los alumnos responden a una breve encuesta individual por escrito que permite múltiples adaptaciones, dependiendo del tipo de asignaturas, del tipo de alumnos, del tipo de información a conseguir, etc.

Debido a los beneficios que nos ha reportado la aplicación de la técnica de "One minute paper" en la clase magistral, proyectamos trasladar esta técnica al entorno virtual. De hecho, hemos programado para el segundo cuatrimestre a punto de comenzar varias encuestas de reflexión, al estilo de "One minute paper", en las que el estudiante responde de forma anónima a dos o tres cuestiones sobre las actividades que se han realizado en el entorno virtual. No sólo pretendemos conseguir información de cómo va marchando el proceso de enseñanza y aprendizaje sino que también tratamos de conseguir que los estudiantes se detengan a reflexionar sobre lo que están aprendiendo, a sintetizar la información que han aprehendido, a relacionar los hechos que ya conocen o a recapacitar sobre su propio proceso de aprendizaje. La utilización de encuestas de reflexión en el entorno virtual permite que el estudiante responda en el momento que más le convenga y eso puede conllevar una meditación más profunda y extensa sobre los asuntos que se le preguntan. 


\section{TUTORÍAS INDIVIDUALES Y COOPERATIVAS: MENSAJES Y FOROS}

La comunicación entre profesor y estudiante y entre los estudiantes es muy rica cuando se trabaja en la enseñanza presencial, pues todos los agentes del proceso educativo están en el mismo lugar y al mismo tiempo en multitud de ocasiones. Y se puede mejorar añadiendo las posibilidades de comunicación y debate que nos ofrecen las plataformas de e-learning. Nosotras estamos usando los mensajes y los foros de Moodle para realizar tutorias proactivas y reactivas.

\section{- Las tutorías individuales: mensajes}

Hay muchas razones, por todos conocidas, que justifican la necesidad de utilizar los mensajes al correo electrónico para complementar la comunicación presencial. Escribimos algunas:

- El estudiante puede mantener un diálogo asíncrono con las profesoras.

- El correo electrónico es un medio de consulta que a veces es más rápido que el presencial (a veces los ca- lendarios de profesor y estudiante son difícilmente compaginables).

- El estudiante puede escribir y enviar un mensaje en el momento que le asalte una duda.

- El estudiante debe tratar de formular con claridad sus ideas o su pregunta.

- El correo electrónico puede ayudar a que el estudiante tímido se atreva a preguntar.

- Se pueden atender casos especiales (enfermedad, trabajo, etc.).

- Durante los períodos lectivos sin clases presenciales, los estudiantes cuya residencia familiar está fuera de Zaragoza necesitan, y de hecho usan, los mensajes como medio de tutoría.

\section{- Las tutorías cooperativas: foros}

Hemos usado dos tipos de foros para las tutorías cooperativas: foros de Pregunta y Respuesta (en adelante, PyR) y los foros abiertos.

Los primeros, los foros PyR, son una modalidad de la actividad foros que Moodle ofrece. Su funcionamiento exige que el estudiante responda al mensaje de inicio del debate enviado por la profesora si quiere ver las contribuciones de

\begin{tabular}{|c|c|}
\hline & Moctrar respuastes ab foma do fia $v$ \\
\hline \multirow[t]{4}{*}{$\rho_{1}$} & 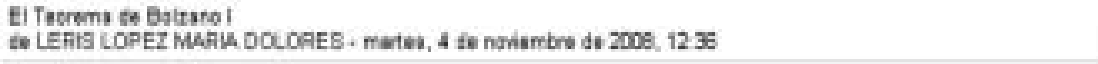 \\
\hline & $\begin{array}{l}\text { Lee le siguerte atrmeción: } \\
\qquad \text { "Una función continua que no se anula en un intervalo, } \\
\text { no cambia de signe en ese interrale" }\end{array}$ \\
\hline & Decide es es cierta o false. \\
\hline & 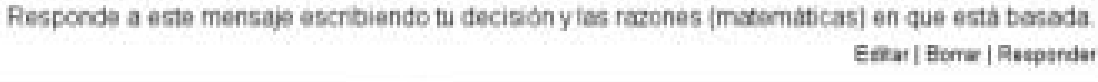 \\
\hline 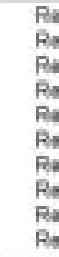 & 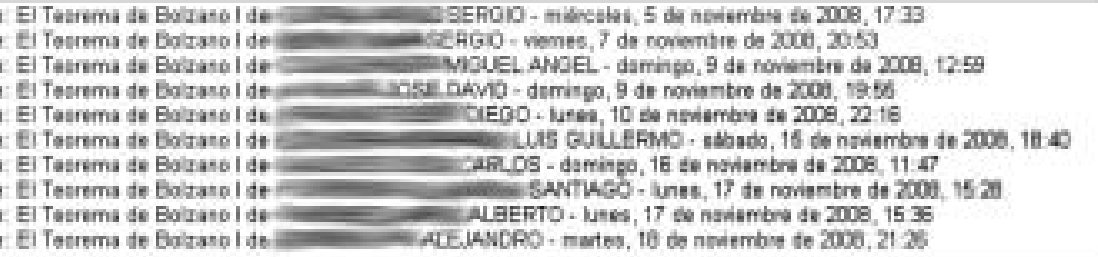 \\
\hline
\end{tabular}

Figura 4. Relación de intervenciones en un foro PyR. 
los demás estudiantes. Estamos usando los foros PyR para tutorías proactivas, es decir, una vez detectados errores o dificultades comunes a través de los cuestionarios, de las tareas $y_{1}$ en general, de las actividades del curso, nos adelantamos a la previsible y esperable consulta de los estudiantes proponiéndoles una pregunta o una explicación incompleta sobre la que debatir dirigida a subsanar la dificultad de aprendizaje detectada.

Se realizaron tutorias grupales y telemáticas reactivas a través de los foros previamente definidos en la plataforma Moodle. De esta forma se fomentó la cooperación entre los alumnos y la conciencia de cómo podian ayudarse entre ellos al hacer públicas sus dudas. Desarrollaron una gran creatividad a la hora de enviar dudas a los foros del curso en el ADD y más teniendo en cuenta que se trata de dudas de matemáticas lo que dificulta enormemente la redacción de los mensajes. Esta situación era crítica especialmente cuando la calidad de la conexión de Internet no era especialmente buena desde sus casas y si el editor de ecuaciones no funcionaba correctamente. Incluso en esos casos no supuso ningún obstáculo, los alumnos participaron y recibieron de buen grado esta iniciativa.

Se detectó una gran cantidad de visitas a los mensajes de los foros y menos participación en ellos en comparación con las visitas. Según sus propias fuentes, el sentido del ridículo es importante a la hora de plantear una duda y no están acostumbrados a resolverse dudas de forma comunitaria.

Por otra parte reconocían que el uso de los foros para dudas es un buen procedimiento ya que les provee de las soluciones a muchas de las dudas surgidas en el tiempo de estudio. Además estás podian ser resueltas de forma asíncrona y de forma virtual. Al principio se mostraron reticentes a realizar tutorías grupales e incluso existía a menudo la figura del "oyente", alumno que no pregunta nada y escucha todas las preguntas y respuestas de sus compañeros. Con el tiempo los "oyentes" pasaron a participar de las sesiones.

La experiencia de estos últimos cursos ha demostrado que los foros como medio de tutoría cooperativa ofrecen ventajas como las siguientes: ayuda a vencer el "pudor" a escribir en público; a pesar de disponer de un editor de ecuaciones, deciden expresar fórmulas y símbolos con texto plano (por ejemplo, suelen utilizar las expresiones de Matlab para las matrices o "trucos" verdaderamente ingeniosos); aprenden a expresar sus dudas concretas y se dan cuenta cuándo preguntan algo demasiado general o abstracto; aprenden mejor los conceptos al intentar explicarlos a sus compañeros; aprenden a expresarse de forma correcta en público (muy pocos utilizan la "jerga" de los móviles y, si lo hacen, sólo al principio); se dan cuenta de que no ponen atención al leer los enunciados de los problemas, los mensajes de los compañeros, etc.; expresan sus opiniones pero siempre con la confianza de que los posibles errores serán subsanados por sus compañeros $y_{\text {, en }}$ caso necesario, por la profesora y alaban el hecho de encontrar resueltas algunas de sus dudas (se ve la conveniencia de ir generando un repositorio de respuestas a las preguntas más frecuentes, las llamadas FAOS).

Y también hemos encontrado desventajas. Por un lado las debidas a las limitaciones de la plataforma como: los foros no permiten organizar los mensajes y aunque se creen varios temas en un foro, dentro de cada uno puede llegar a reinar el caos o el sistema de respuesta y anidado de los mensajes puede conducir a un desorden absoluto. Todo ello desanima a los alumnos que acceden tarde al curso, pues se sienten "abrumados" por la cantidad de mensajes y eso les retrae a participar. Por otro lado, es necesario establecer unas normas de conducta para que las intervenciones en los foros sean realmente útiles; por ejemplo, es necesario que el asunto de los mensajes sea suficientemente explicativo, pues en caso contrario se pueden leer asuntos como "ejercicio" o "problema" lo que, de ninguna manera, expresa el contenido del mensaje o también conviene advertir a los estudiantes de que los contenidos de sus mensajes deben ser concretos y centrados en una sola duda.

En resumen, es muy recomendable dar instrucciones claras al principio del curso sobre el uso de los foros indicando: cómo crear el asunto de un mensaje nuevo, cómo responder, cómo cambiar el asunto de un mensaje aunque sea de respuesta y cómo recopilar mensajes. Suele ser muy ilustrativo mostrar algunos ejemplos de malas prácticas y confrontarlos con modelos de las buenas prácticas de cursos académicos anteriores. 


\section{Conclusiones}

Los alumnos agradecen cualquier iniciativa que les induzca, de alguna forma, a llevar un ritmo de trabajo continuo, y que les permita comprobar sus niveles de conocimiento en cada momento. Su participación en las actividades suele ser entusiasta pero, con el avance del curso, algunos van abandonándolas al no conseguir, en general, mantener el ritmo de trabajo de todas las asignaturas. Esta observación pone en el punto de mira un aspecto esencial para el éxito del proyecto de cambio metodológico que se propugna en el EEES: la coordinación entre las asignaturas de un mismo curso académico para que, entre otras cosas, el esfuerzo total del estudiante para superar el curso completo no le desborde.

Las debilidades mencionadas en este artículo, como el hábito de estudio, son obstáculos que asoman a lo largo de todo el proyecto de aprendizaje. También se han ido poniendo de manifiesto algunas carencias en su formación previa en cuanto a habilidades y conocimientos matemáticos. La constatación de esta situación fue el desencadenante de dos actuaciones del grupo FMI en el que han intervenido y siguen interviniendo las autoras: evaluar el perfil de ingreso de un estudiante a los grados de ingeniería (Riaguas, Arribas, Celorrio y Lerís, 2006; Boal y otros, 2007; Bueno y otros, 2008 y Boal, Bueno, Leris y Sein-Echaluce, 2008-2), crear un entorno de aprendizaje virtual (Sein-Echaluce y otros 2008) y diseñar un curso de iniciación a la formación en competencias matemáticas.

Los estudiantes recién ingresados en la Universidad están acostumbrados a realizar su trabajo de forma individual, suelen desconocer las ventajas del trabajo en grupo y algunos tienden a prejuzgarlo como una pérdida de tiempo. La puesta en práctica de unos pocos trabajos en grupo con la atención y asesoramiento de la profesora suele ser el mejor remedio contra esos prejuicios. Podemos contar en el haber de nuestra experiencia que se ha conseguido fomentar el trabajo cooperativo, que los estudiantes mejoran su capacidad de comunicar matemáticas y que, en definitiva, han mejorado su conocimiento de algunos asuntos matemáticos y han desarrollado sus habilidades sociales.
Recibido: 20 de marzo de 2009

Aceptado: 16 de abril de 2009

\section{AGRADECIMIENTOS}

Este trabajo ha sido realizado en el marco del proyecto TSI2005-04127 financiado por el Ministerio de Educación y Ciencia, España.

\section{BIBLIOGRAFÍA}

Bará, J. y Valero, M. (2007): Técnicas de aprendizaje cooperativo. Notas de los autores del curso impartido en el Instituto de Ciencias de la Educación de la Universidad de Zaragoza, marzo de 2007.

Bartolomé, A. (2004): Blended learning. Conceptos básicos. Universidad de Barcelona. PixelBit. N. ${ }^{2} 23$. Abril.

www.Imi.ub.es/personal/bartolome/articuloshtml/04_blended_learning/docu- mentacion/1_bartolome.pdf (última consulta el 30/01/2009).

Boal, N., Leris, D. y Sein-Echaluce, M. L. (2008-3): Una experiencia de trabajo colaborativo para potenciar el desarrollo de las destrezas matemáticas. Actas del XVI Congreso Universitario de Innovación Educativa de las Enseñanzas Técnicas (CUIEET), Cádiz, España.

Boal, N., Bueno, C., Leris, D. y Sein-Echaluce, M. L. (2008-2): "Las habilidades matemáticas evaluadas en las Pruebas de Acceso a la Universidad. Un estudio en varias universidades públicas españolas", Revista de Investigación Educativa, vol. 26-1 (2008), pp. 11-23.

Boal, N., Arribas, M., Lerís, D. y Sein-Echaluce, M. L. (2008-1): Curso de Orientación a las Matemáticas en Ingeniería. II Jornadas de Innovación Docente, 
Tecnologías de la Información y la Comunicación e Investigación Educativa en la Universidad de Zaragoza, febrero de 2008. Zaragoza, Spain.

Boal, N., Bueno, C., Castelló, J., Lerís, D. y Martínez, V. (2007): Expectations And Realities On The Mathematical Formation Of The Students Of New Access To The University. INTED2007 Proceedings of International Technology, Education and Development Conference IATED. Valencia, Spain.

Boal, N. y Sein-Echaluce, M. L. (2006): Una experiencia en la Enseñanza de las Matemáticas. I Jornadas de Innovación Docente, Tecnologías de la Información y la Comunicación e Investigación Educativa en la Universidad de Zaragoza, noviembre de 2006. Caminando hacia Europa, p. 50. Cap. I-21. Documento: 11 páginas. ISBN: 978-84-96214-85-9.

Bueno, C., Lerís, D., Boal, N., Castelló, J., Correas, J.M., Martínez, V. y SeinEchaluce, M. L. (2008): Assessment of Attitudes and Mathematical Skills for First Year University Students. INTED2008 Proceedings of International Technology, Education and Development Conference IATED. Valencia, Spain. ISBN: 978-84-6120190-7.
Creed, T. (1996): "Think-Pair-Share-DISCUSS" published in Cooperative Learning and College Teaching, vol. 7, no. 1, http:// employees.csbsju.edu/TCREED/tpsd. html (última consulta 30/01/2009).

Guzmán, M. (2003): Cómo hablar, demostrar y resolver en Matemáticas. Madrid, España: Editorial Anaya.

Instructional Strategies Online (2006): Saskatoon Public School Division http://olc.spsd.sk.ca/DE/PD/instr/ strats/think/index.html (última consulta 30/01/2009).

Leris, D., Boal, N., Bueno, C. y Sein-Echaluce, M. L. (2008): El Valor de las Competencias Matemáticas en las Pruebas de Acceso a la Universidad. Actas del 16cuieet, Cádiz, España.

Moodle. www.moodle.org.

OCDE (2004): Project PISA: Learning for tomorrow's world (2004).

Real Decreto 938/2001, de 3 de agosto, en el que se establece el currículo de $B a-$ chillerato (BOE de 7 de septiembre de 2001).

Riaguas, A., Arribas, M., Celorrio, R. y Leris, D. (2006): El acceso a los estudios de Ingeniería: detección de debilidades o carencias formativas en Matemáticas, Actas del 4. ${ }^{\circ}$ Congreso Internacional de Docencia Universitaria e Innovación, $\mathrm{Cl}$ DUI. Barcelona, España, 5 al 7 de julio.
Ross, K. P., Angelo, T. (2001): The OneMinute-Paper. Resource: Classroom Assessment Techniques. http://www. csupomona.edu/ biology/teaching_ bytes/one-minute-paper.pdf (última consulta 30/01/2009).

Sein-Echaluce, M. L., Boal, N. y Leris, D. (2008): B-Learning to Promote Cooperative-Tutorial Activities in Scientific Subjects. ED-MEDIA 2008, Viena, Austria.

Sein-Echaluce, M. L., Martínez, V., Lerís, D., Correas, J. M., Castelló, J., Boal, N. (2008): An e-Learning Platform Project for International University Cooperation. INTED2008 Proceedings of International Technology, Education and Eevelopment IATED. Valencia, Spain. ISBN: 978-84-612-0190-7.

Stead, D. R. (2005): "A review of the oneminute paper", Active Learning in Higher Education, vol. 6, no. 2, pp. 118-131. http://alh.sagepub.com/cgi/ content/abstract/6/2/118 (última consulta 30/01/2009).

Valero, M. (2009): Evaluación de competencias genéricas en los programas formativos de las ingenierías. Notas del autor del curso impartido en el Instituto de Ciencias de la Educación de la Universidad de Zaragoza, enero de 2009. 